\title{
Polynomial based Channel Estimation Technique with Sliding Window for $M$-QAM Systems
}

\author{
O. O. Ogundile*, M. O. Oloyede ${ }^{\dagger}$, F. A. Aina ${ }^{\ddagger}$ and S. S. Oyewobi ${ }^{\S}$ \\ *Department of Physics and Telecommunications, Tai Solarin University of Education Ijagun, Ogun State, Nigeria \\ †Department of Information and Communication Science, University of Ilorin, Ilorin, Kwara State, Nigeria \\ †Department of Telecommunication Science, University of Ilorin, Ilorin, Kwara State, Nigeria

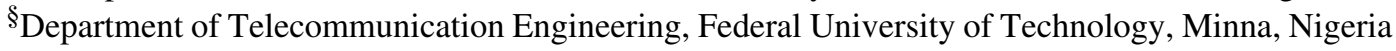

\begin{abstract}
Pilot Symbol Assisted Modulation (PSAM) channel estimation techniques over Rayleigh fading channels have been analysed in recent years. Fluctuations in the Rayleigh fading channel gain degrades the performance of any modulation scheme. This paper develops and analyses a PSAM Polynomial interpolation technique based on Least Square $(L S)$ approximations to estimate the Channel State Information (CSI) for $M$-ary Quadrature Amplitude Modulation ( $M$-QAM) over flat Rayleigh fading channels. A Sliding window approach with pilot symbol adjustment is employed in order to minimize the computational time complexity of the estimation technique. The channel estimation performance, and its computational delay and time complexity is verified for different Doppler frequencies $\left(f_{d}\right)$, frame lengths $(L)$, and Polynomial orders ( $P$-orders). Simulation results show that the Cubic Polynomial interpolation gives superior Symbol Error Rate (SER) performance than the Quadratic Polynomial interpolation and higher $P$-orders, and the performance of the Polynomial estimation techniques degrade with increase in the $P$-orders.
\end{abstract}

Keywords-Channel estimation; Doppler frequency; frame length; interpolation; polynomial order

\section{INTRODUCTION}

Channel estimation assuming a Rayleigh fading channel with Additive White Gaussian Noise (AWGN) is an essential aspect of wireless communication. Most especially if Orthogonal Frequency Division Multiplexing (OFDM) or $M$ ary Quadrature Amplitude Modulation ( $M$-QAM) is adopted as the modulation scheme in conveying information between the transmitter and receiver. Rayleigh channel gain variations result in degradation in the performance of the modulation scheme, more significantly at low Signal-to-Noise Ratio (SNR) [1]. Therefore, channel estimation is required to obtain a rough estimate of the Channel State Information (CSI), and compensate for such channel errors.

Pilot Symbol Assisted Modulation (PSAM) channel estimation techniques have been proposed in literature using Gaussian, Wiener, Sinc, Linear interpolations, and many more interpolation techniques to analyse and compensate for the effect of fading errors in order to improve the performance of the modulation scheme [2]-[8]. The major focus has been on the trade-off between the computational time complexity and gain performance of these channel estimation techniques over Rayleigh fading channels.

This paper proposes a Polynomial interpolation channel estimation technique based on Least Square $(L S)$ approximations for $M$-QAM assuming a flat Rayleigh fading channel. The Polynomial estimation technique offers a balance between the performance and implementation time complexity in estimating the CSI. Simulations are provided to verify the optimal performance of the interpolation technique for different Polynomial orders $(P$-orders $)$, while increasing the Doppler frequency $f_{d}$, and frame length size $L$. Result is also shown comparing the developed Polynomial interpolation technique with some existing channel estimation techniques found in [4], [7] and [9]. The simulation results are shown assuming rectangular 16-QAM, although the estimation technique can be extended to other $M$-QAM schemes as well.

The remainder of this paper is structured as follows. Section II gives a brief explanation of the system model and notations. In section III, the PSAM Polynomial interpolation technique is developed and analysed for different P-orders. Discussions and result comparisons are presented in Section IV. Finally, the paper is summarised and concluded in Section $\mathrm{V}$.

\section{System Model}

Fig. 1 shows a pilot symbol assisted modulation system model. The input data is mapped to rectangular 16-QAM complex data symbols with the real and imaginary components taken from the set $( \pm 1 c, \pm 3 c, \ldots \pm(m-1) c)$, where $m$ is of the form $M=m^{2}$ [10]-[12]. Pilot symbols are periodically inserted to the transmitted 16-QAM complex data symbols. The transmitted QAM symbols are divided into frames of size $L$, with each frame starting with a known symbol called the pilot symbol, and subsequently $L-1$ data symbols. The frame structure is transmitted over a flat Rayleigh fading channel with Doppler frequency $f_{d}$.

After down-converting and matched filtering, the faded received signal corrupted with AWGN in the current $p$-th frame is defined as:

$$
\bar{r}_{p}^{l}=\bar{E}_{p}^{l} \bar{t}_{p}^{l}+N_{p}, \quad l=1, \ldots, L-1,
$$




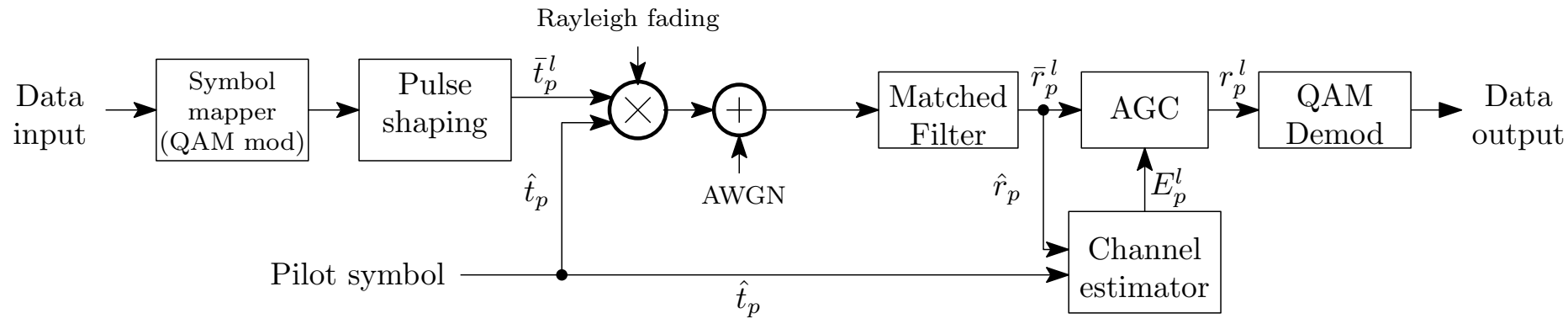

Fig. 1: PSAM system model.

where $N_{p}$ is the complex AWGN with variance $N_{o} / 2, \bar{t}_{p}^{l}$ is the transmitted QAM symbols, and $\bar{E}_{p}^{l}$ is the complex zero mean Gaussian variables denoting the fading distortion at the data symbol positions.

Similarly, the faded received pilot symbol corrupted with AWGN in the current $p$-th frame is defined as:

$$
\hat{r}_{p}=\hat{E}_{p} \hat{t}_{p}+N_{p},
$$

where $\hat{E}_{p}$ is the complex zero mean Gaussian variables denoting the fading distortion at the pilot symbol positions, $\hat{t}_{p}$ and $\hat{r}_{p}$ are the transmitted and received pilot symbols respectively. Thus, the noisy fading estimates at the $p$-th pilot symbol positions is obtained as:

$$
E_{p}=\hat{E}_{p}+\frac{N_{p}}{\hat{t}_{p}} .
$$

The fading estimates $E_{p}^{l}$ in the current $p$-th frame at the data symbol position is obtained by interpolating the pilot symbol fading estimates $E_{p}$. The received data symbol $\bar{r}_{p}^{l}$ is scaled (dividing the received data symbol $\bar{r}_{p}^{l}$ by the estimated fading distortion $E_{p}^{l}$ ) to compensate for the channel error in a process called Automatic Gain Control (AGC). The scaled received data symbol $r_{p}^{l}$ is demodulated to obtain a copy of the transmitted data.

\section{PSAM POLYNOMIAL InTERPOLATION}

The Polynomial interpolation channel estimation technique maintains a balance between the performance and implementation time complexity in estimating the CSI. The fading distortion in the current $p$-th frame at the data symbol position is estimated by interpolating the current $p$-th frame pilot symbol fading estimate $E_{p}$ and the nearest pilot symbol fading estimates $E_{p+i}$ to the $p$-th frame. The number of the nearest pilot symbol estimates used for the interpolation process depends on the P-order.

Consider the complex data and pilot symbols frame structure at the output of the matched filter as shown in Fig. 2. Given that $\left(E_{p}, E_{p+1}, \ldots, E_{p+i}\right)$ are the nearest pilot symbol fading estimates to the l-th data symbol and $\left(K_{p}, K_{p+1}, \ldots, K_{p+i}\right)$ are the known positions of the pilot symbols in the received frame structure of Fig. 2. The fading distortion is computed in the current $p$-th frame as:

$$
E_{p}^{l}=\sum_{l=1}^{L-1} \gamma_{1}(l)^{0}+\gamma_{2}(l)^{1}+\ldots+\gamma_{i+1}(l)^{i}
$$
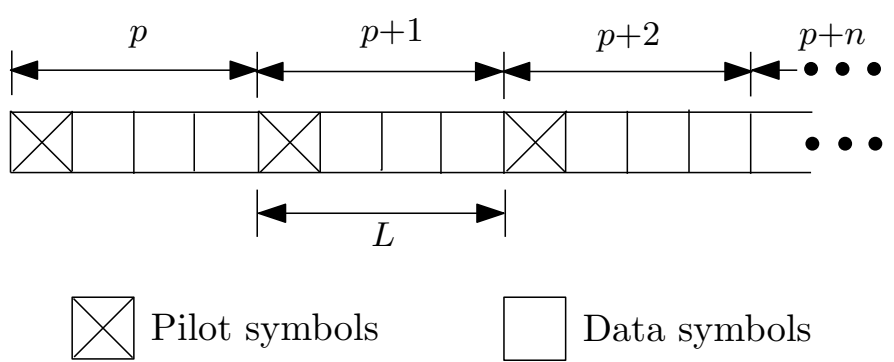

Fig. 2: Data and pilot symbols frame structure.

where $i$ is the $P$-order, and $\left(\gamma_{1}, \gamma_{2}, \ldots, \gamma_{i+1}\right)$ are the Polynomial interpolation coefficients which is define as:

$$
\left[\begin{array}{c}
E_{p} \\
E_{p+1} \\
\vdots \\
E_{p+i}
\end{array}\right]=\left[\begin{array}{cccc}
1 & K_{p} & \ldots & K_{p}^{i} \\
1 & K_{p+1} & \ldots & K_{p+1}^{i} \\
\vdots & \vdots & \ddots & \vdots \\
1 & K_{p+i} & \ldots & K_{p+i}^{i}
\end{array}\right]\left[\begin{array}{c}
\gamma_{1} \\
\gamma_{2} \\
\vdots \\
\gamma_{i+1}
\end{array}\right] \text {. }
$$

This estimation technique experiences delay with increase in the frame length size $L$ but the computational time complexity remains the same. Thus, the computational time complexity of the Polynomial interpolation technique is independent of $L$. However, as the P-order increases, the estimation technique experiences delay and the computational time complexity increases at a linear rate, $(O(n)) \rightarrow\left(O\left(n^{i}\right)\right)$. In order to reduce the computational time complexity of this estimation technique from $\left(O\left(n^{i}\right)\right) \rightarrow\left(O\left(n^{i-1}\right)\right)$, a sliding window approach is assumed in computing the Polynomial interpolation coefficients.

The nearest pilot symbol positions are fixed as $\left(K_{1}, K_{1+L}, \ldots, K_{1+i L}\right)$, while the fading estimates at the pilot symbol positions are shifted from $E_{p}$ to $E_{p+1}$ when computing the Polynomial interpolation coefficients from frame $p$ to $p+n$. Therefore, Eqn. 5 is modified as:

$$
\left[\begin{array}{c}
E_{p} \\
E_{p+1} \\
\vdots \\
E_{p+i}
\end{array}\right]=\left[\begin{array}{cccc}
1 & 1 & \ldots & 1 \\
1 & K_{1+L} & \ldots & K_{1+L}^{i} \\
\vdots & \vdots & \ddots & \vdots \\
1 & K_{1+i L} & \ldots & K_{1+i L}^{i}
\end{array}\right]\left[\begin{array}{c}
\gamma_{1} \\
\gamma_{2} \\
\vdots \\
\gamma_{i+1}
\end{array}\right] .
$$

The transmitted frame structure starts with a pilot symbol and subsequently $L-1$ data symbols, so $K_{1}$ is always equal 


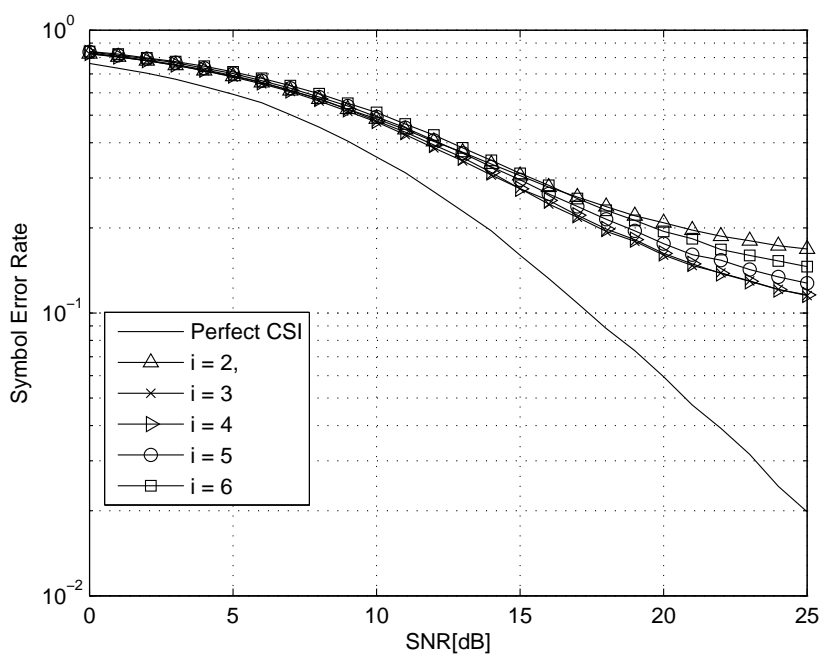

(a) Frame length $L=4$ and Doppler frequency $f_{d}=200 \mathrm{~Hz}$

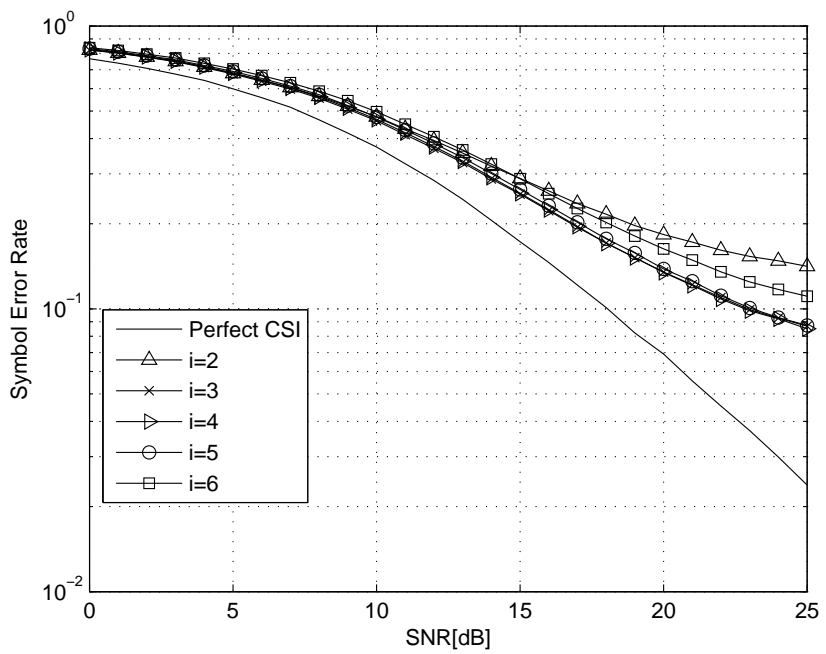

(c) Frame length $L=8$ and Doppler frequency $f_{d}=100 \mathrm{~Hz}$

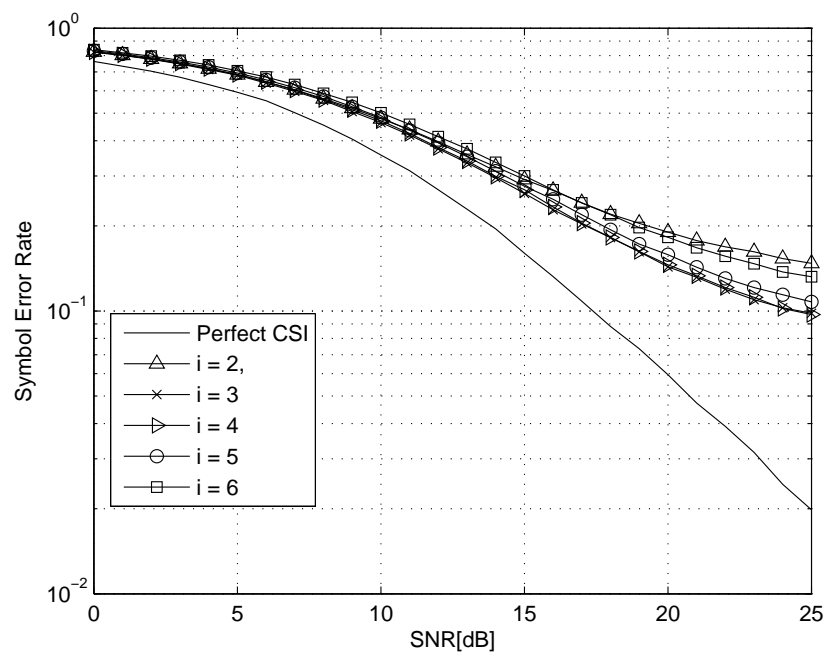

(b) Frame length $L=6$ and Doppler frequency $f_{d}=150 \mathrm{~Hz}$

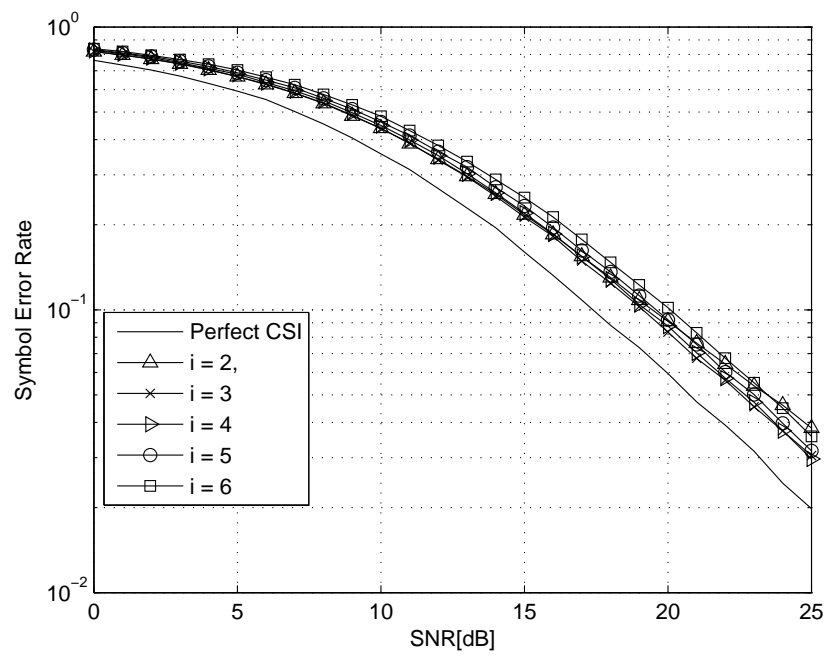

(d) Frame length $L=10$ and Doppler frequency $f_{d}=50 \mathrm{~Hz}$

Fig. 3: SER performance comparison for different $P$-orders $i$ and Doppler frequencies $f_{d}$ assuming rectangular 16-QAM over flat Rayleigh fading channels.

to one $\left(K_{1}=1\right)$. Applying Eqns. 6 and 4, computing the fading estimates at the data symbol positions in the current $p$-th frame is straightforward.

\section{Results AND Discussion}

The performance analysis of the Polynomial based channel estimator is carried out assuming a 16-QAM scheme over flat Rayleigh fading channel. The estimator is applied for different values of Doppler frequency $f_{d}$; from $50 \mathrm{~Hz}$ which denotes a practical slow-varying flat Rayleigh fading channel to a fast-varying flat Rayleigh fading channel $(100 \mathrm{~Hz}-200 \mathrm{~Hz})$. The performance of the polynomial channel estimator is also tested for increase in $P$-order and frame length size $L$ as shown in Fig. 3 , in order to verify the optimal $P$-order for real time channel estimations. Note that a different frame length size $L$ is used as the Doppler frequency is increased from $50 \mathrm{~Hz}$ to $200 \mathrm{HZ}$. This is because the performance of most channel estimator plummets as $L$ increase. Thus, in order to achieve a reasonable Symbol Error Rate (SER) performance for higher Doppler frequency, a smaller $L$ is used in the simulation set-up as $f_{d}$ increases.

The Polynomial estimator provides a practical computational time complexity of $\left(O\left(n^{i-1}\right)\right)$ to the overall system model of Fig. 1 for large input data size. This implies that the computational time complexity of the Polynomial estimator increases with increase in the P-order. The Polynomial estimator provides a practical computational time complexity of $\left(O\left(n^{i-1}\right)\right)$ to the overall system model of Fig. 1 for large input data size. This implies that the computational time complexity of the Polynomial estimator increases with increase in the $P$-order.The Polynomial estimator provides a practical computational time complexity of $\left(O\left(n^{i-1}\right)\right)$ to the overall system model of Fig. 1 for large input data size. This implies that the computational time complexity of the Polynomial estimator 
increases with increase in the P-order.The Polynomial estimator provides a practical computational time complexity of $\left(O\left(n^{i-1}\right)\right)$ to the overall system model of Fig. 1 for large input data size. This implies that the computational time complexity of the Polynomial estimator increases with increase in the $P$ order. Thus, the Quadratic polynomial interpolator $(i=2)$ offers the minimum computational time complexity compared to all other $P$-orders $(2 \leq i \leq \infty)$, and it experiences the lowest computational delay.

However, as shown in Fig. 3, the Cubic polynomial interpolator $(i=3)$ yields better SER performance compared to the Quadratic interpolator, and higher Polynomial interpolator orders. It is also observed from Fig. 3 that as the P-order increases from $i=3 \rightarrow i=6 \rightarrow i=\infty$, the SER performance of the Polynomial interpolator degrades, and the computational delay and time complexity increases as well. The Cubic interpolator gives a SER gain of $+1.5 d B$ over the Quadratic interpolator at SNR $>15 d B$ as shown in Fig. 3(a)-(c), and it offers moderate computational time complexity. Hence, the Cubic polynomial estimator provides a balance between the performance of the channel estimation technique and its implementation time complexity. The Cubic estimator therefore produces the "optimal" performance among all Polynomial estimation technique of the form of Eqns. 6 and 4 over flat Rayleigh fading channels.

The optimal performance of the Polynomial channel estimation technique $(i=3)$ is compared with two existing channel estimation techniques in literature. Fig 4. shows the SER performance of the Cubic estimator with the Sinc interpolator proposed be Kim et al. [4], and the Linear interpolator analysed in [7] and [9]. The estimators are applied assuming a fast-varying flat Rayleigh fading channel (Doppler frequency $f_{d}=100 \mathrm{~Hz}$ ), and for frame length sizes $L=4$ and $L=6$. The Cubic interpolator yields a significant SER performance of $+1.5 \mathrm{~dB}$ over the Linear, and Sinc channel estimators at SNR $>15 d B$. Also, Fig. 4 shows that the Cubic interpolator at frame length $L=6$ outperforms the Sinc, and Linear interpolators at frame length $L=4$. Although, the proposed Polynomial estimation technique has the price of increased computational time complexity compared to the Sinc, and Linear estimators, it is a useful channel estimation technique and can be implemented for real time systems over Rayleigh fading channels.

\section{Conclusion}

A pilot symbol assisted modulation Polynomial channel estimation technique has been developed and analysed assuming rectangular 16-QAM over a flat Rayleigh fading channel. The performance of the Polynomial channel estimation technique is tested for different P-orders, while increasing the Doppler frequency $f_{d}$ and frame length $L$ in order to find out the practical optimal performance of the proposed estimator. The estimator technique attains its optimal performance at $P$-order $i=3$, whereby it maintains a balance between the performance of the channel estimation technique and its computational time complexity. The optimal performance $(i=3)$ of the Polynomial channel estimation technique gives better SER performance than the Sinc, and Linear estimators over fast flat Rayleigh fading channel. It is noted that the developed Polynomial channel interpolator evinces considerably higher computational time complexity compared to the Sinc, and Linear interpolators;

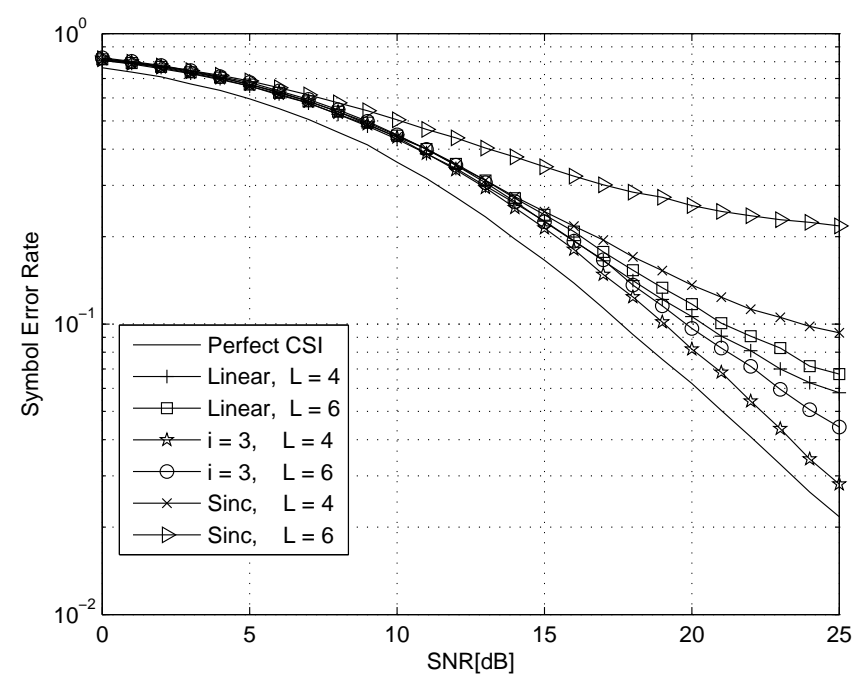

Fig. 4: SER performance comparison for the Cubic, Sinc, and Linear estimators assuming rectangular 16-QAM over a fast flat Rayleigh fading channel. Doppler frequency, $f_{d}=100 \mathrm{~Hz}$.

however, it offers significant SER performance gain and can be implemented for real time systems. Results provided in Fig. 3 and 4 affirm these analysis.

\section{REFERENCES}

[1] Theodore S. Rappaport, Wireless communications: principles and practice. Prentice Hall PTR, 2nd edition, September 2002.

[2] J. Cavers, "An Analysis of Pilot Symbol Assisted Modulation for Rayleigh Fading Channels." IEEE, Transaction on Vehicular Technology, vol. 40, no. 4, pp. 686-693, November 1991.

[3] S. Sampei and T. Sunaga, "Rayleigh Fading Compensation for QAM in Land Mobile Radio Communications." IEEE ,Trans. Veh. Technol., vol. 42, pp. 137-146, May 1993.

[4] Y. Kim, G. Jeong, Y. Bang, H. Park, and S. Choi, "New Rayleigh Fading Channel Estimator Based on PSAM Channel Sounding Techniques." IEEE, vol. 3, pp. 1518-1520, 1997.

[5] X. Tang, M. Alouini, and A. Goldsmith, "Effect of Channel Estimation Error on $M$-QAM BER Performance in Rayleigh Fading." IEEE,Trans. Commun., vol. 47, pp. 1856-1864, December 1999.

[6] S. Coleri, M. Ergen, A. Puri, and A. Bahai, "Channel Estimation Techniques Based on Pilot Arrangement in OFDM Systems," IEEE Transactions on Broadcasting, vol. 48, no. 3, September 2002.

[7] M. Benjillahi and L. Szczeciński, "Low Complexity Channel Estimation with Pilot Symbol Assisted Modulation.” IEEE, Signal Processing and Its Applications, vol. 2, pp. 471-474, August 2005.

[8] Y. Shen and Ed Martinez, "Channer Estimation in OFDM Systems," freescale semiconductor, AN3059, January 2006.

[9] A. Mämmelä and V. Kaasila, "Smoothing and Interpolation in a PilotSymbol Assisted Diversity System." International Journal of Wireless Information Networks, vol. 4, no. 3, 1997.

[10] M. K. Simon and J. G. Smith, "Carrier Synchronization and Detection of QASK Signal Sets," IEEE, Transactions on Communication, vol. 22, no. 2, pp. 98-106, February 1974.

[11] O. Ogundile and D. Versfeld, "Improved reliability information for rectangular 16-QAM over flat rayleigh fading channels," in Computational Science and Engineering (CSE), 2014 IEEE 17th International Conference on, Dec 2014, pp. 345-349.

[12] _ " "Improved reliability information for OFDM systems on timevarying frequency-selective fading channels," in Wireless Telecommunications Symposium (WTS), 2015, April 2015, pp. 1-7. 